(2) Open Access Full Text Article

\title{
A network pharmacology approach to determine the synergetic mechanisms of herb couple for treating rheumatic arthritis
}

This article was published in the following Dove Press journal:

Drug Design, Development and Therapy

\author{
$X i-x i X u^{\prime}$ \\ Jian-ping $\mathrm{Bi}^{2}$ \\ Li Ping ${ }^{3}$ \\ Ping $\mathrm{Li}^{\prime}$ \\ Fei $\mathrm{Li}^{1,4}$
}

'State Key Laboratory of Natural Medicines, China Pharmaceutical University, Nanjing, People's Republic of China; ${ }^{2}$ Orthopedics Department, Shandong Provincial Traditional Chinese Medical Hospital, Affiliated Hospital of Shandong University of Traditional Chinese Medicine, Jinan, People's Republic of China; ${ }^{3}$ Center for Drug Safety Evaluation and Research, Zhejiang University, Hangzhou, People's Republic of China; ${ }^{4}$ School of Pharmacy, Xinjiang Medical University, Urumqi, People's Republic of China
Correspondence: Fei Li; Ping Li State Key Laboratory of Natural Medicines, China Pharmaceutical University, No 24 Tongjia Lane, Nanjing 210009 , People's Republic of China

Tel/fax +86 258327 I 382

Email lifeicpu@।63.com; liping2004@।26.com
Purpose: The purpose of this study was to investigate the therapeutic mechanism(s) of Clematis chinensis Osbeck/Notopterygium incisum K.C. Ting ex H.T (CN).

Methods: A network pharmacology approach integrating prediction of ingredients, target exploration, network construction, module partition and pathway analysis was used.

Results: This approach successfully helped to identify 12 active ingredients of $\mathrm{CN}$, interacting with 13 key targets (Akt1, STAT3, TNFsf13, TP53, EPHB2, IL-10, IL-6, TNF, MAPK8, IL-8, RELA, ROS1 and STAT4). Kyoto Encyclopedia of Genes and Genomes pathway enrichment analysis indicated that $\mathrm{CN}$-regulated pathways were mainly classified into signal transduction and immune system.

Conclusion: The present work may help to illustrate the mechanism(s) of action of CN, and it may provide a better understanding of antirheumatic effects.

Keywords: targets prediction, pathways analysis, action mechanism, Clematis chinensis Osbeck, Notopterygium incisum K.C. Ting ex H.T. Chang

\section{Introduction}

$\mathrm{RA}$ is a chronic autoimmune disease influenced by genetic factors, environmental factors and interaction. ${ }^{1}$ The prevalence of RA is $\sim 1 \%$ in the adult population, with a higher incidence in the elderly and women. ${ }^{2}$ In addition to disability and joint destruction, ${ }^{3}$ patients with RA have a higher risk of dying prematurely from cardiovascular diseases. ${ }^{4}$ Consequently, prevention and treatment of RA are critical in clinical therapy. Therapeutic agents for RA include NSAIDs, glucocorticoids, DMARDs, biologic DMARDs and, most recently, small molecular signal inhibitors. ${ }^{5}$ However, most of current drugs, which play an important role in treating RA, have severe adverse effects, including gastrointestinal irritation, kidney injury, cardiovascular risk and even the so-called Cushing's syndrome. ${ }^{6}$ Consequently, TCMs, with clinical application for thousands of years, have recently attracted more and more attention due to prominent effectiveness and less side effects. ${ }^{7} \mathrm{CC}$ and NI, as TCMs, have been frequently used to treat RA for their anti-inflammatory activity. ${ }^{8-10}$ Our previous study has reported that $\mathrm{CN}$ has evident anti-rheumatic effects in adjuvant-induced arthritis in rats. ${ }^{11}$ However, the molecular mechanism(s) of CN in the treatment of RA remains to be elucidated. Network pharmacology is an efficient tool to clarify targets and mechanisms of TCMs. ${ }^{12}$ The methodologies of network pharmacology highlight the paradigm shift from "one drug, one target" to "multicomponent therapeutics, biological network". ${ }^{13}$ TCMs have the advantages of multiple components and targets, which 
correspond to the methodologies of network pharmacology. Thus, network pharmacology is desirable for exploring the mechanisms of TCMs. In the present study, we respectively collected the information of targets from active ingredients in $\mathrm{CN}$ and targets of RA from several databases for the first time. In order to uncover the rationality of $\mathrm{CN}$, network construction and topological structural analysis were established, which offered underlying synergistic mechanisms of $\mathrm{CN}$ for treating RA.

\section{Methods}

\section{Building database of ingredients}

All the chemical ingredients' data of CC and NI were derived from Traditional Chinese Medicine Systems Pharmacology Database and Analysis Platform (TCMSP) (http://sp.nwu. edu.cn/tcmsp.php). TCMSP is a unique system pharmacology platform of TCMs that is capable of providing the relationship between drugs, targets and diseases.

\section{Screening of active ingredients}

The active constituents from $\mathrm{CC}$ and NI were filtered by integrating $\mathrm{OB}$ and DL. DL helps to describe pharmacokinetic and pharmaceutical properties of compounds, such as solubility and chemical stability. Usually, the selection criterion for the "drug-like" compounds in TCMs is $0.18 .{ }^{14}$ OB represents the relative amount of an oral drug that is absorbed into the blood circulation. ${ }^{15}$ Since low OB is the primary reason responsible for the development of TCMs into therapeutic drugs, it is vital to conduct OB screening criterion. Based on literatures and suggestions in TCMSP, we selected $\mathrm{OB} \geq 30 \%$ and $\mathrm{DL} \geq 0.18$ as a screening threshold. ${ }^{16,17}$ The ingredients conforming to both standards mentioned earlier will be preserved for further analysis.

\section{TCM-associated target prediction}

Three databases are combined to predict relevant targets of active ingredients in CC and NI comprehensively. GeneCards database (http://www.genecards.org/) automatically integrates gene-centric data from $\sim 125$ web sources, while BATMAN-TCM (http://bionet.ncpsb.org/batman-tcm) ranks potential drug-target interactions based on their similarity to the known drug-target interactions. STITCH database (http:// stitch.embl.de/) integrates many sources of experimental and manually curated evidence with text-mining information and interaction predictions. First, the active constituents were severally entered into GeneCards, BATMAN-TCM and STITCH. Then, duplications and unified names were removed from the targets obtained from the aforementioned three tools. Noteworthy, only the targets of Homo sapiens were kept for further study.

\section{RA-associated target prediction}

Different genes associated with RA were collected from DisGeNET (http://www.disgenet.org/web/DisGeNET). DisGeNET is a useful platform providing the search of the molecular underpinnings of diseases, the analysis of disease genes, the validation of predicted genes and so on.

\section{Network construction and node screening}

The different targets from CC, NI and RA were submitted to Agilent Literature Search 3.1.1 (LitSearch version 2.69). Based on the human targets, we set "Max Engine Matches" as 10 and searched through the whole text. Then, the protein-protein interaction network was visualized by Cytoscape 3.5.1 software. Finally, we severally selected the top 30 targets of high-node degree as key targets for further analysis.

\section{Module partition and KEGG pathway analysis}

MCODE was applied to identify the molecular network for module identification according to the clustering of genes in the network. Then, main modules obtained from CC, NI and RA were submitted to DAVID Bioinformatics Resources 6.8 software (https://david.ncifcrf.gov/) to carry out GO functional enrichment analysis. "Homo Sapiens" was also limited to identify KEGG pathways that were significantly enriched in the identification module. Of note, $P$-value was implemented to explore the statistical significance of the modules. KEGG pathways with $P<0.05$ ( $P$-values were corrected using the Benjamini-Hochberg procedure) are significant signaling pathways.

\section{Results}

\section{Active ingredients of $\mathrm{CC}$ and $\mathrm{NI}$}

A total of 484 ingredients of $\mathrm{CN}$ were retrieved from TCMSP, including 114 ingredients of CC and 370 ingredients of NI. In this study, 21 active compounds from 484 compounds met both the requirements, $\mathrm{OB} \geq 30 \%$ and $\mathrm{DL} \geq 0.18$ (Table 1 ). It has been validated experimentally that some ingredients possess pharmacological activities. For example, $\beta$-sitosterol $(\mathrm{OB}=36.91, \mathrm{DL}=0.75)$ plays a 
Table I Active ingredients of CC and NI

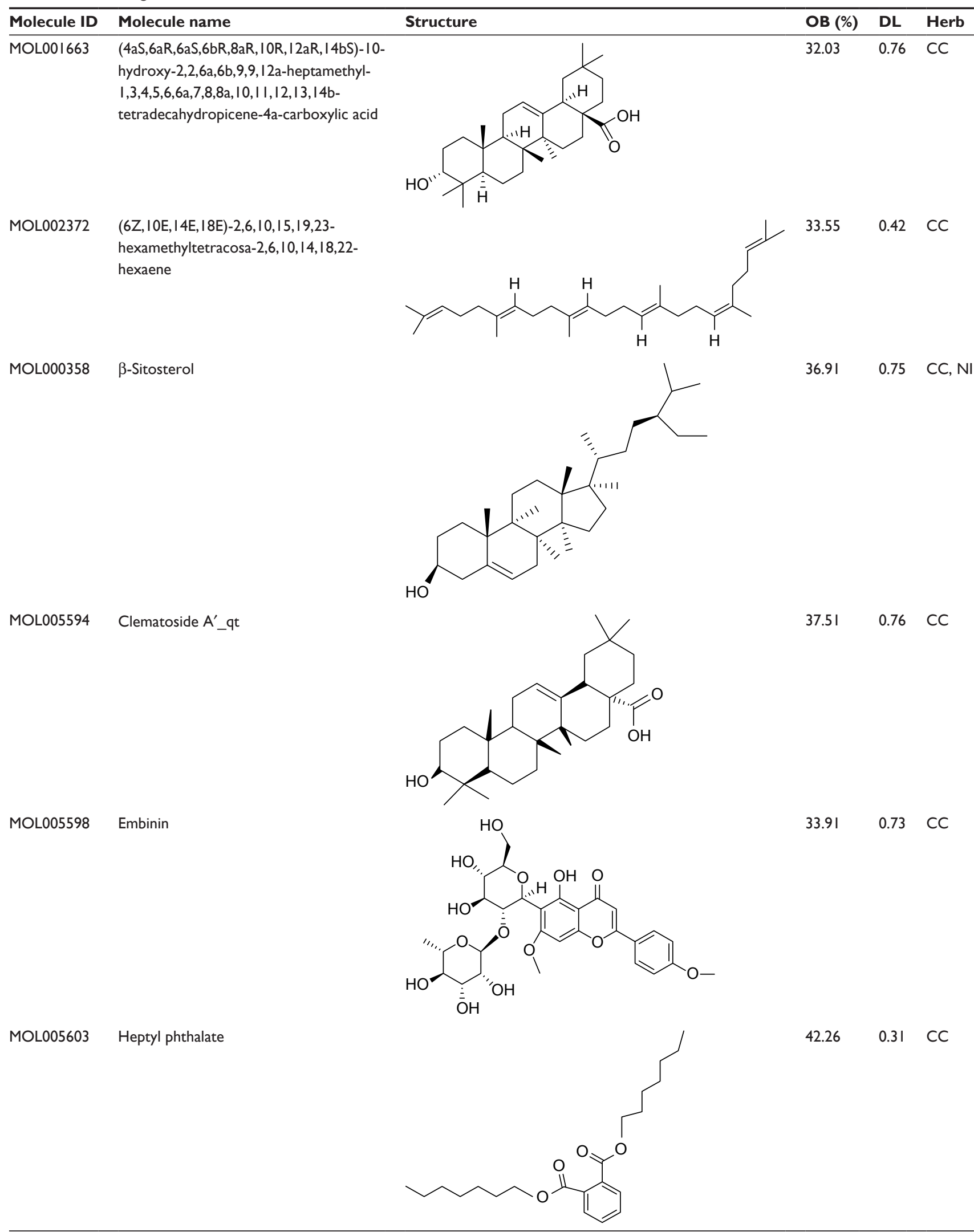

(Continued) 
Table I (Continued)

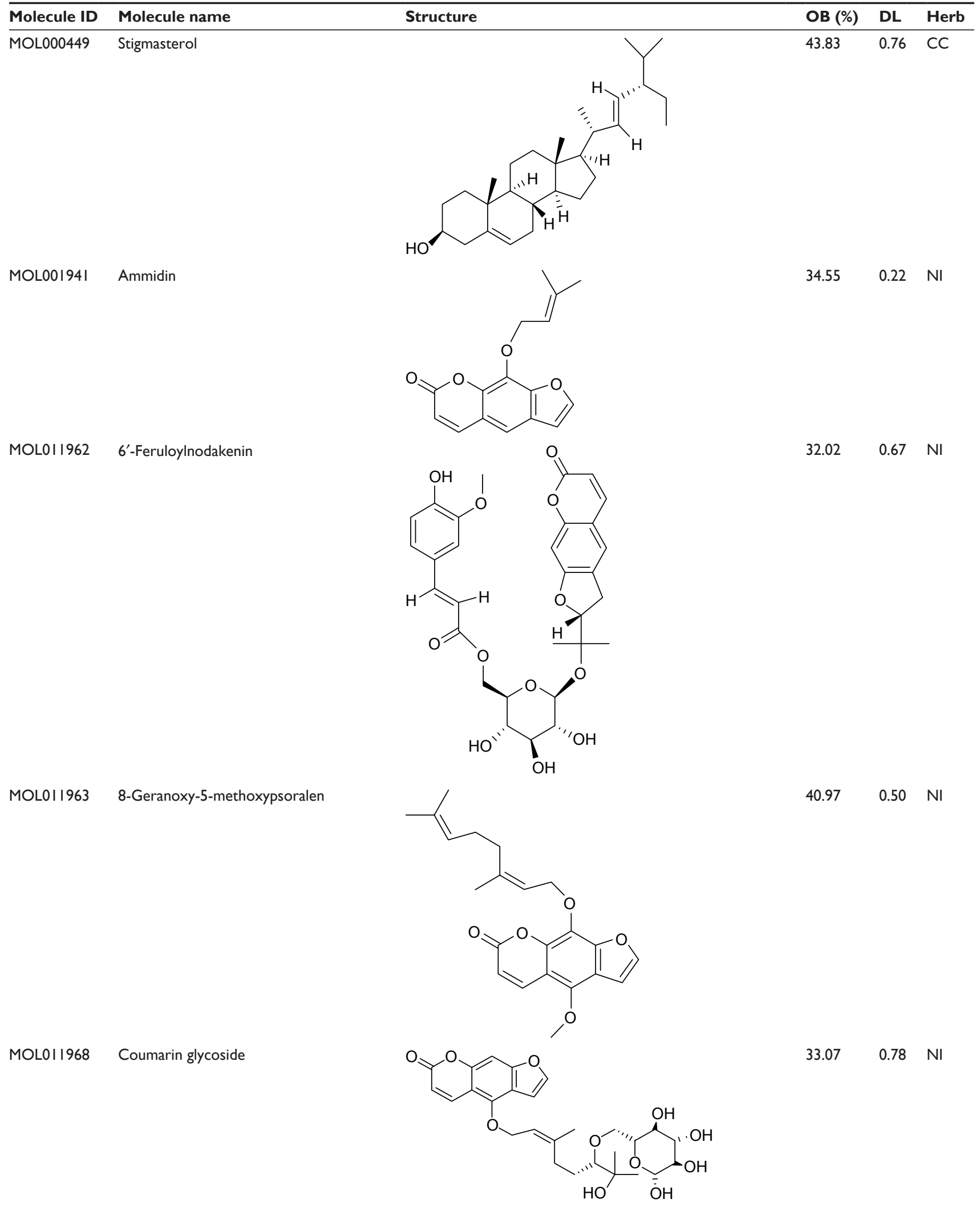

(Continued) 
Table I (Continued)

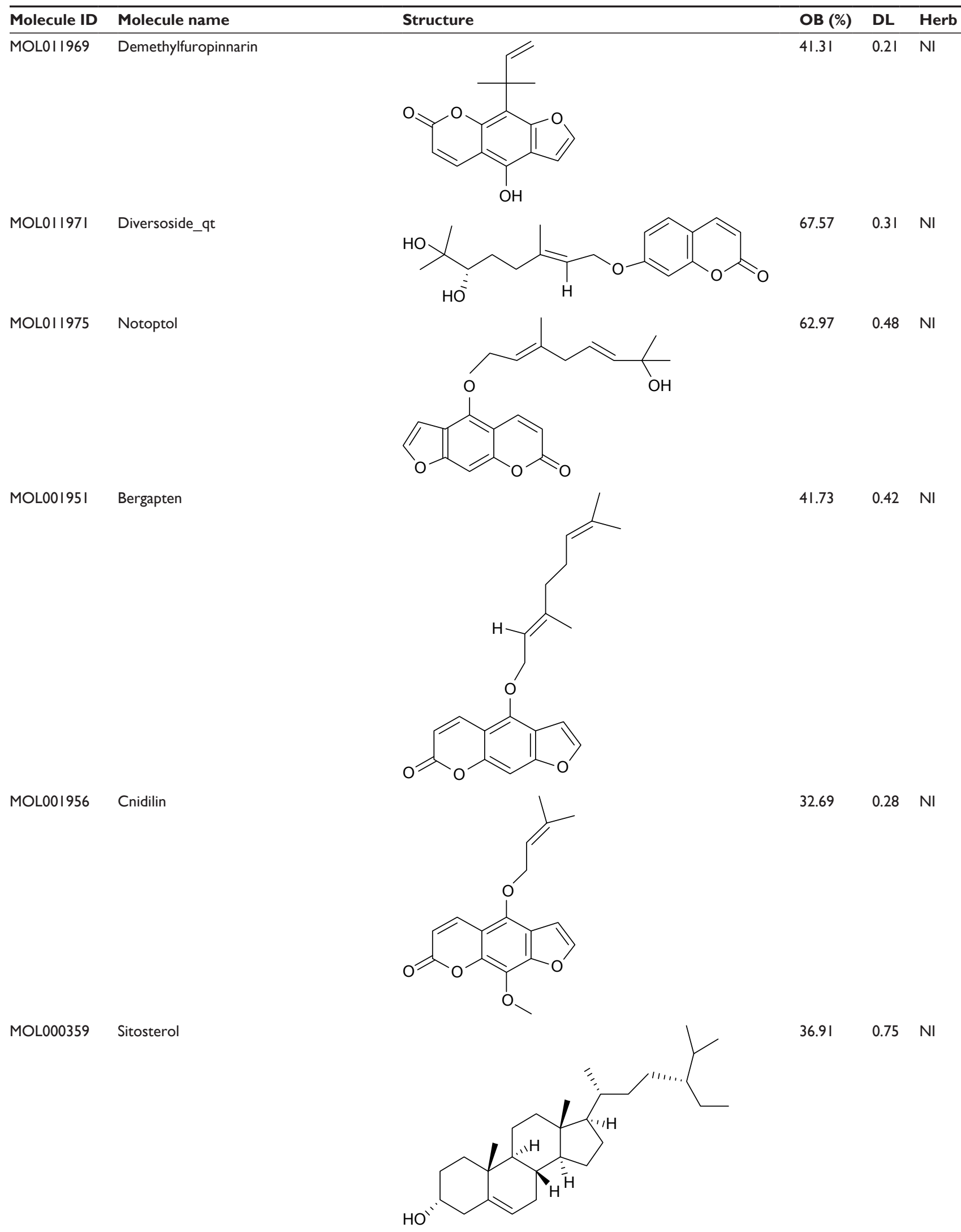

(Continued) 
Table I (Continued)

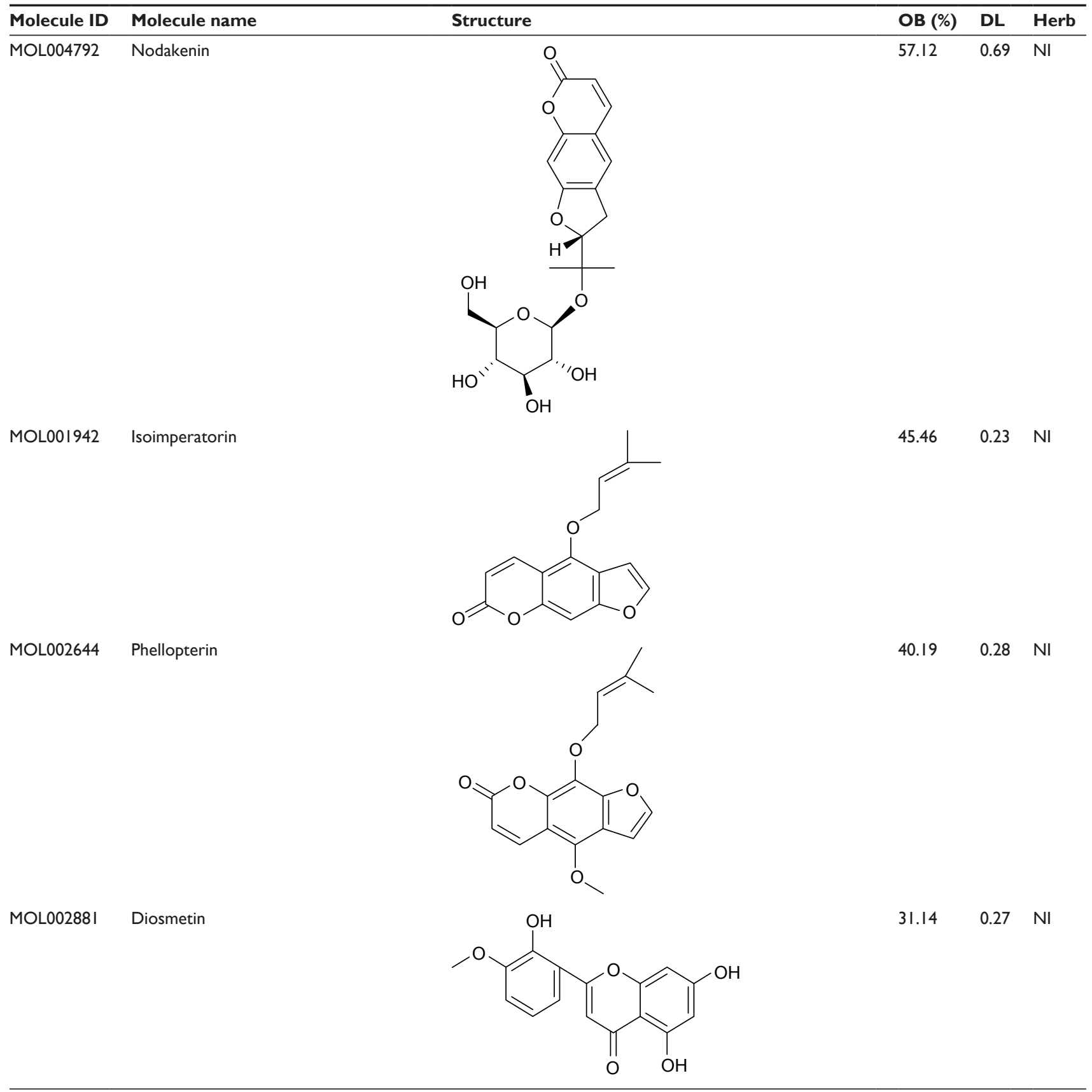

Abbreviations: CC, Clematis chinensis Osbeck; NI, Notopterygium incisum K.C. Ting ex H.T. Chang; OB, oral bioavailability; DL, druglikeness.

significant role in anti-inflammation, ${ }^{18}$ anti-tumor ${ }^{19}$ and anti-hyperlipidemia. ${ }^{20}$ Moreover, nodakenin $(\mathrm{OB}=57.12$, $\mathrm{DL}=0.69$ ) plays an important therapeutic effect in inflammatory disorders and has been regarded as one of the standard ingredients of NI in Chinese Pharmacopoeia. ${ }^{10,21}$

\section{Target prediction}

TCMs give play to their pharmacological effects through multiple ingredients and targets. Thus, besides predicting ingredients, it is also necessary for the exploration of targets. However, searching for targets through literatures is time consuming and labor intensive. In the present work, predictive models including GeneCards, BATMAN-TCM and STITCH were used to predict 301 targets, which interacted with 12 active ingredients. It is interesting that another nine ingredients were removed for having no relevant targets. In addition, the DisGeNET database was also used to predict 1,869 targets associated with RA. 


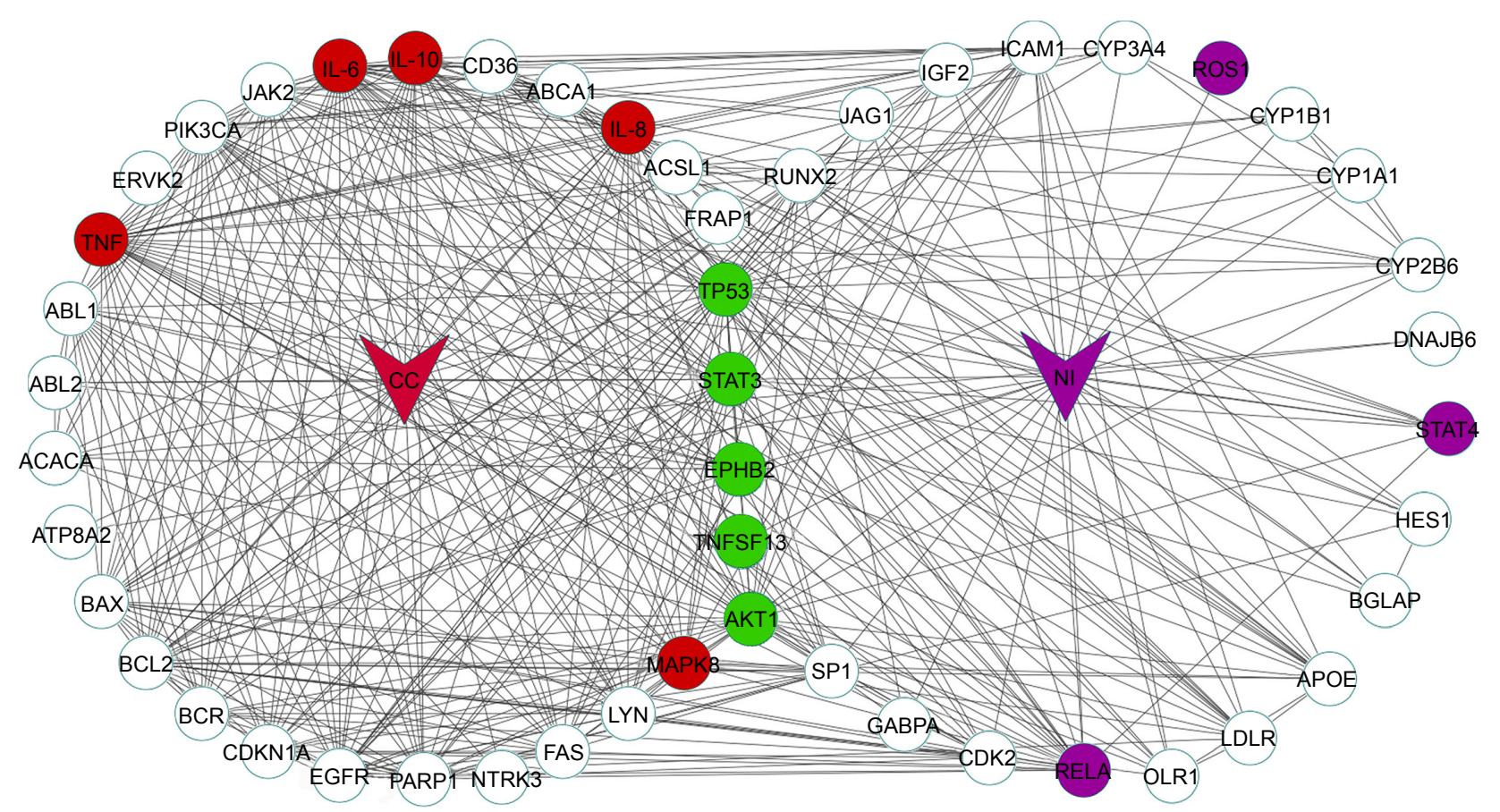

Figure I Top 30 targets of $\mathrm{CC}$ and NI.

Note: Green nodes are shared targets from RA and herb couple, red ones are shared targets from RA and CC and purple ones are shared targets from RA and NI. Abbreviations: CC, Clematis chinensis Osbeck; NI, Notopterygium incisum K.C. Ting ex H.T. Chang; RA, rheumatoid arthritis.

\section{Network construction and node screening}

Network construction was automatically performed after searching by Agilent Literature Search. Molecular network of CC, NI and RA separately consisted of 781,267 and 746 nodes and was connected by 2,873, 633 and 2,303 edges, respectively. The topological parameters of CC, NI and RA show that node-degree distribution obeys the power law distribution. To further investigate the synergistic mechanisms of herb couple, the top 30 targets of high-node degree were severally chosen. As shown in Figure 1, the herb couple shares 13 targets (Akt1, STAT3, TNFsf13/APRIL, TP53, EPHB2, IL-10, IL-6, TNF, MAPK8/JNK, IL-8, RELA, ROS1 and STAT4) with RA, in which five targets (STAT3, Akt1, TP53, TNFSF13 and EPHB2) are the overlapped targets in CC and NI and another five targets (IL-10, IL-6, TNF, MAPK8 and IL-8) and three targets (RELA, ROS1 and STAT4) are separate in CC and NI.

\section{Module partition and KEGG pathway analysis}

Module partition

MCODE software was used to analyze the molecular network. A total of 122 modules were identified from original networks with 47 modules from CC, 25 modules from NI and 50 modules from RA. Top 10 modules with more nodes were respectively selected for KEGG pathway analysis.

\section{KEGG pathway analysis}

In order to deduce the potential pathways affected by herb couple, DAVID Bioinformatics Resources 6.8 software was used to perform pathway enrichment analysis. Since diseases arise from the dysfunctions of basic biological functions, we removed the KEGG pathway section of human diseases. We found that herb couple could totally affect 35 signal pathways, including 16 pathways from both CC and NI, 17 pathways solely from CC and 2 pathways solely from NI.

As shown in Table 2A, herb couple acts on six pathways in signal transduction, such as PI3K-Akt signaling pathway and JAK-STAT signaling pathway. Immune system and endocrine system, respectively, cover three pathways such as NOD-like receptor signaling pathway and GnRH signaling pathway. Furthermore, the herb couple also regulates other pathways in cell process, development and nervous system. Signal pathways solely from CC are shown in Table 2B and indicated that $\mathrm{CC}$ has the possibility of being associated with the immune system, signal transduction, endocrine system and cell process. Compared with the signal pathways in CC, NI solely regulates cytosolic DNA-sensing pathway and cell process, which are associated with signal transduction and cell process, respectively (Table $2 \mathrm{C}$ ).

\section{Discussion}

RA is a chronic autoimmune disease that is implicated in inflammation, angiogenesis, bone destruction and the 


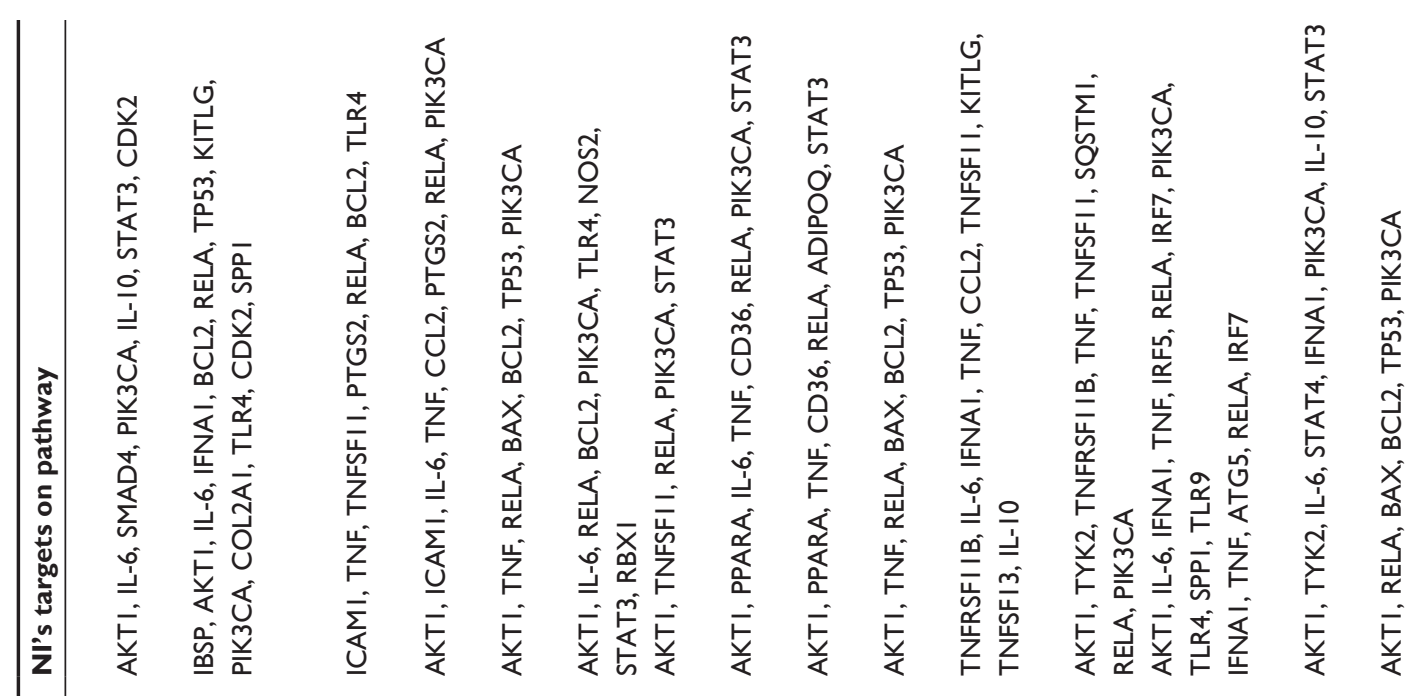

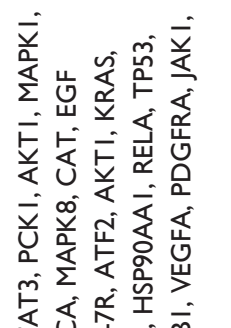

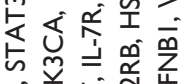

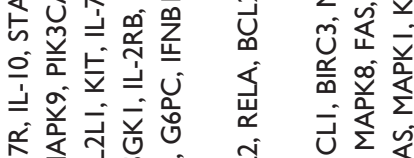

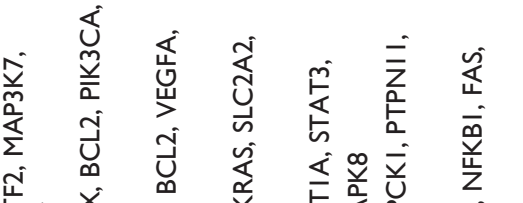

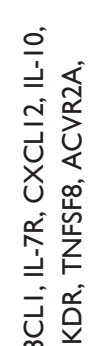

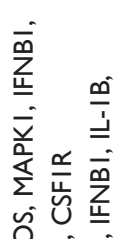

过茛它

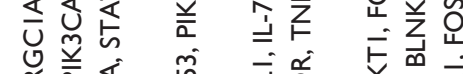

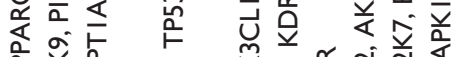

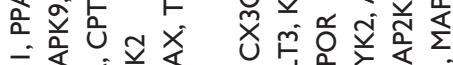

商衣过这余

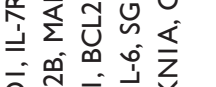

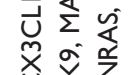

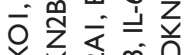

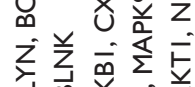

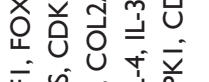

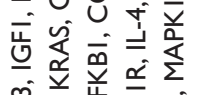

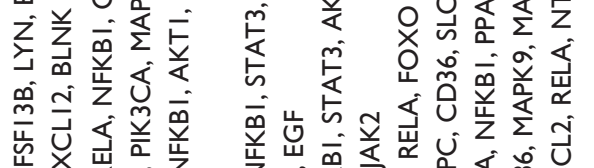

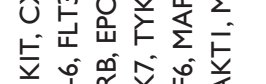

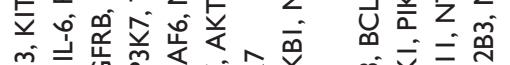

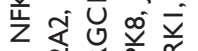

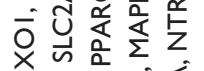

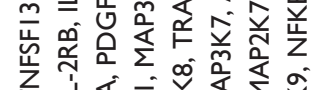

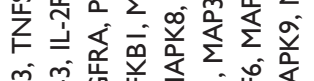

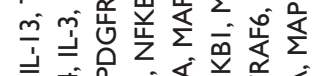

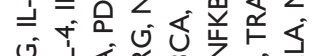

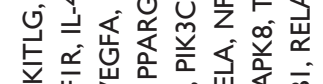

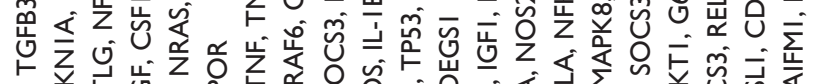

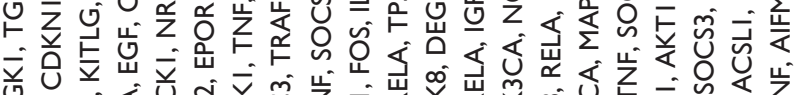

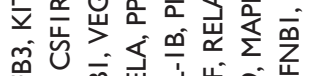

这远的

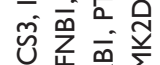

O

安安

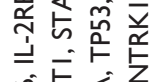

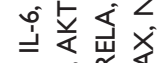

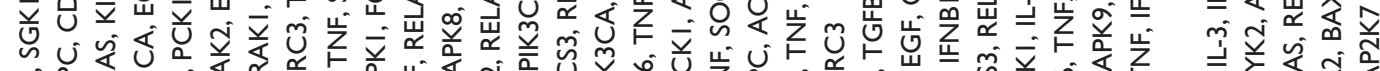

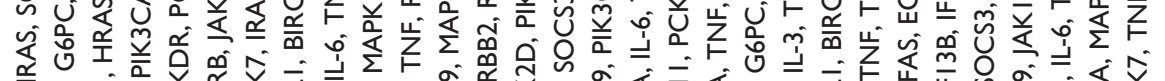

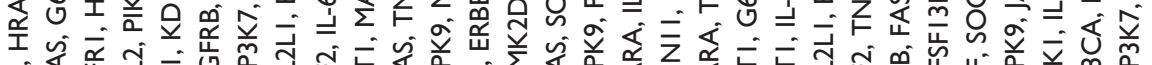

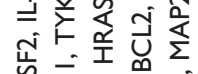

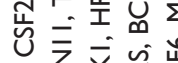

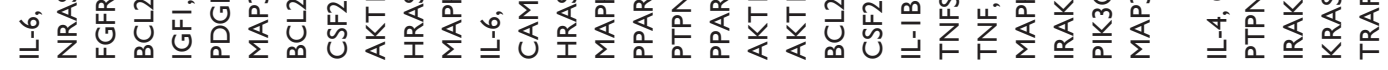
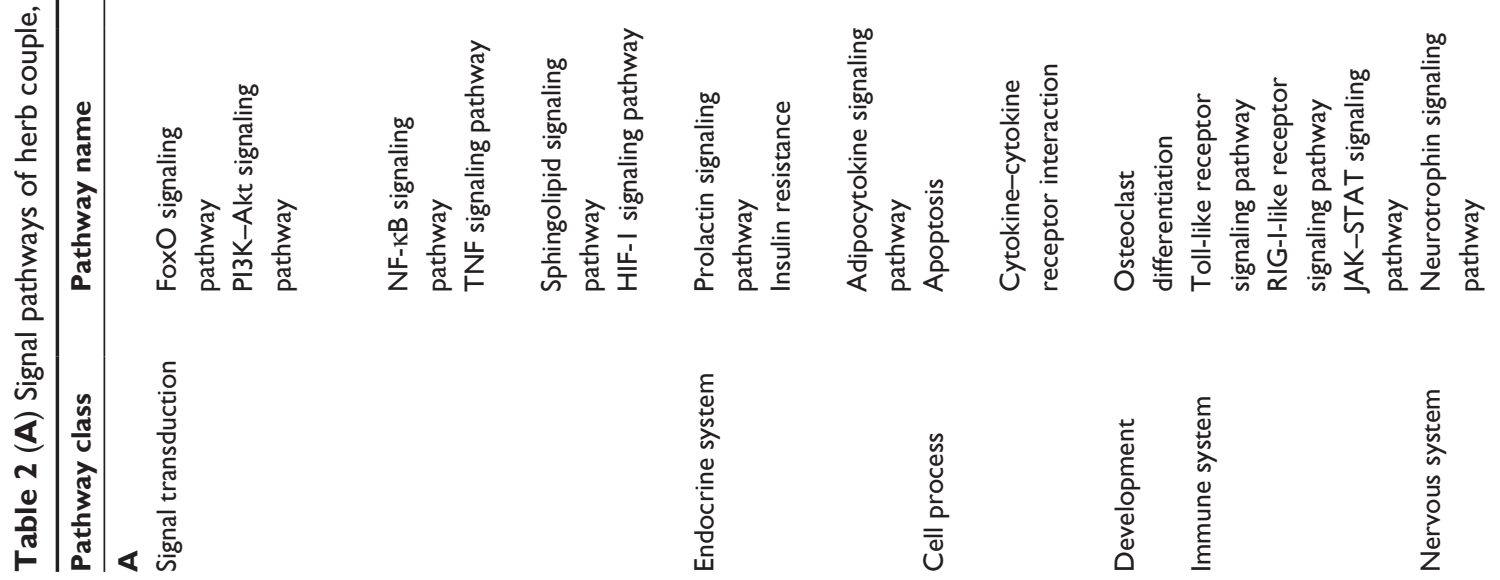

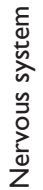



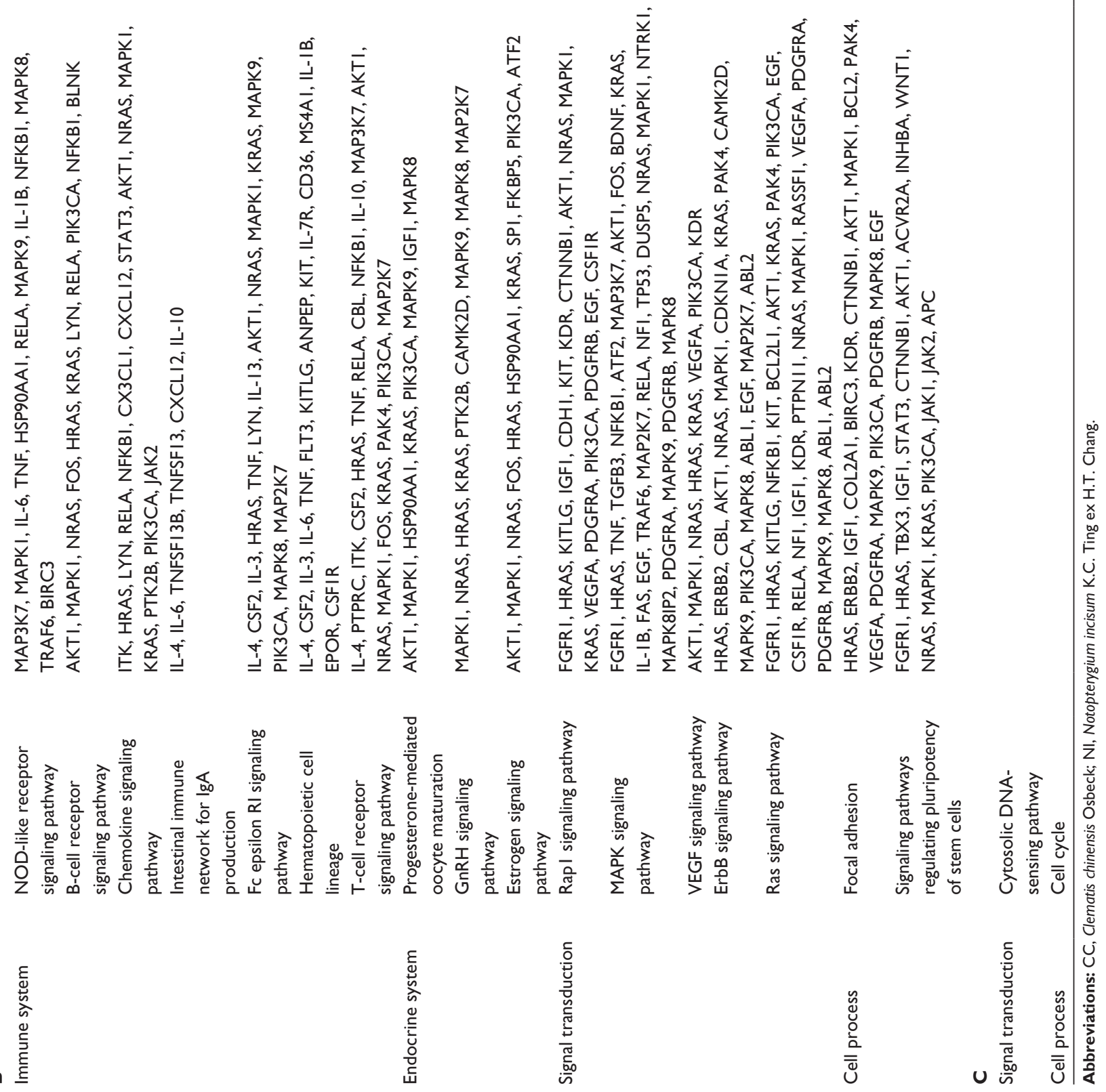
immune regulation. Therapeutic agents for RA, including NSAIDs, glucocorticoids and DMARDs, are limited due to their side effects. ${ }^{22}$ TCMs are common drugs for the treatment of RA, with clinical effectiveness and less adverse effects. Our prior study has noted that herb couple of CC and NI was experimentally validated possessing antirheumatic effects. ${ }^{11}$ However, TCM, as a multi-component synergistic system agent, is comprehensive and abstruse. Therefore, their research method is different from chemical drugs. In the present study, to better recognize the drug combination of $\mathrm{CN}$, we proposed a network pharmacology approach integrating prediction of ingredients and pathway analysis strategy of targets for $\mathrm{CN}$. The method was applied to explore the potential regulation of inflammatory response, immune system and angiogenesis of $\mathrm{CN}$ and provide a new sight for the treatment of RA. Nonetheless, our method still has some limitations and needs to further improve. The approach just predicts and analyzes the potential synergetic mechanism of CC and NI from the perspective of biological network. Clinical and experimental trials are required to be further validated.

\section{Targets analysis of herb couple} Targets related to RA

Synovial inflammation is a basic pathological change in RA and results in swelling and pain in the joints of RA patients. Thus, anti-inflammation is critical in the treatment of RA. Results of key targets' analysis found that RA and $\mathrm{CN}$ shared a total of 13 targets. Among these targets, IL-6, IL-8 and IL-10 all belonged to the IL family. In RA, IL-6 can be released by monocytes, macrophages and endothelial cells and influences T-cell development, which indirectly promotes the production of Th1, Th2 and Th17 cells with proinflammatory properties. IL-6 can also increase the level of VEGF in synovial fibroblasts, aggravating joint inflammation and damage. ${ }^{23}$ In addition to inflammation, IL-6 increases osteoclast recruitment by acting on hematopoietic stem cells, leading to joint damage in RA. ${ }^{24,25}$ Therefore, blockade of IL-6 action is effective to reduce both inflammation and joint destruction in RA. ${ }^{26}$ The anti-inflammatory response is essential to control the degree and duration of the inflammatory response in RA. In macrophages, the anti-inflammatory response relies on IL-10/JAK/STAT3 signaling pathway. IL-10 signaling cascade starts upon IL-10 binding to IL-10R and activates STAT3 via the JAK1 kinase. ${ }^{27}$ STAT3 stimulates the transcription of specific genes and in turn represses proinflammatory cytokines such as IL-1, IL-6, IL-12 and TNF- $\alpha$. Moreover, MAPK8, also known as JNK, is activated in RA synovium and mediates joint destruction in adjuvant arthritis of rats. ${ }^{28}$ MAPK8 signalosome represents a target to prevent joint destruction. ${ }^{29}$ TNFSF13 sustains B-cell activation and thus enhances autoimmune diseases. It also regulates synovial inflammation in RA. ${ }^{30}$ Therefore, TNFSF13 could also be a therapeutic strategy aimed at downregulating synovial inflammation. Furthermore, other targets are also involved in the process of RA, such as STAT4, ${ }^{31}$ EphB2, ${ }^{32}$ TP53, ${ }^{33} \mathrm{Akt}^{34}$ and RELA. ${ }^{35}$ Upregulation or downregulation of abovementioned targets contributes to treating RA. On the other hand, ROS1, highly expressed in a variety of tumor cell lines, is used as a drug target to suppress tumors clinically. ${ }^{36}$ Based on our predictions, we speculate that ROS1 may have some relevance with RA, and the result would be validated in our future study.

\section{Pathway analysis of herb couple Pathways related to immune response}

As shown in Table 2, many signal pathways are classified into immune system and regulate the balance of the immune system. Innate immune is the first line of defense against foreign pathogens and is the basis and initiator of adaptive immunity. B and T cells are well known to be related to adaptive immune response. ${ }^{37}$ Table $2 \mathrm{~B}$ shows that $\mathrm{CC}$ is associated with B-cell receptor signaling pathway and T-cell receptor signaling pathway, indicating that CC may have a play in the adaptive immune response. In addition, we also found that $\mathrm{CN}$ could regulate some proinflammatory molecule-involved pathways such as chemokine signaling pathway and Fc epsilon RI signaling pathway. In addition, cytosolic DNAsensing pathway, MAPK signaling pathway and apoptosis are highly associated with the function of immune response, although not classified into the immune system. ${ }^{38,39}$ Considering the effects of immune pathways on disease progress and joint destruction, modulation of these pathways may have important implications for treating RA.

\section{Pathways related to inflammation}

Another large category of signal pathways is signal transduction, including a number of well-known signal pathways that are related to inflammation, such as JAK-STAT signaling pathway, NF- $\mathrm{KB}$ signaling pathway and TNF signaling pathway. For example, canonical NF- $\kappa$ B signaling pathway is critical for the regulation of the inflammation response. Although less extensively studied, non-canonical pathway plays an indirect role in synovial inflammation via the high expression of its activators such as CD40L, CD40, BAFF/BAFF-R and RANKL in RA synovium. ${ }^{40}$ Since both 
canonical and non-canonical NF- $\kappa \mathrm{B}$ signaling pathways participate in inflammatory response and the pathogenesis of RA, inhibitors of these two pathways can play a role in the treatment of RA. TNF, as the upstream target of the NF- $\kappa B$ pathway, has already been regarded as a therapeutic target in RA. ${ }^{41}$ Moreover, the JAK-STAT pathway is an important pathway for the transduction of cytokines associated with RA and is regarded as a target in inflammatory and autoimmune diseases. ${ }^{42}$ Tofacitinib, a JAK inhibitor, proves effective in the treatment of RA through reducing the expression of metalloproteinase and interferon-regulated gene in RA synovium. ${ }^{43}$

\section{Pathways related to angiogenesis}

Angiogenesis is a complex process involving the growth of new blood vessels and plays an important role in the growth, metastasis and prognosis of tumor. It is accompanied by the entire process of RA and can foster the infiltration of inflammatory cells into the joints, leading to synovial hyperplasia and progressive bone destruction. ${ }^{44}$ Ras signaling pathway and VEGF signaling pathway regulated solely by $\mathrm{CC}$ are related to angiogenesis. VEGF signaling pathway plays an important role in promoting the proliferation of vascular endothelial cells and the formation of new blood vessels, ${ }^{45,46}$ while Ras signaling pathway is related to tumor angiogenesis and vascular permeability. ${ }^{47}$ Certainly, inhibiting VEGF signaling is a feasible antiangiogenic and anti-inflammatory therapeutic strategy in RA. ${ }^{48}$

\section{Conclusion}

TCMs usually exert a multicomponent and multi-pathway synergetic efficacy in the treatment of various diseases. Therefore, the research approach applied to TCMs should correspond to the mechanisms of synergy. In this study, we applied a network pharmacology approach to identify the RA-related targets and signal pathways of $\mathrm{CN}$, making it possible to connect genomic space to pharmacological space. In summary, we predicted the action mechanism(s) of herb couple for treating RA through the analysis of key targets and KEGG pathways.

\section{Abbreviations}

RA, rheumatoid arthritis; CC, Clematis chinensis Osbeck; NI, Notopterygium incisum K.C. Ting ex H.T. Chang; CN, Clematis chinensis Osbeck/Notopterygium incisum K.C. Ting ex H.T. Chang; TCM, traditional Chinese medicine; NSAIDs, nonsteroidal anti-inflammatory drugs; DMARDs, diseasemodifying antirheumatic drugs; TCMSP, traditional Chinese medicine systems pharmacology database and analysis platform; OB, oral bioavailability; DL, druglikeness; KEGG, Kyoto Encyclopedia of Genes and Genomes; Akt1/PKB, protein kinase B; TNFsf13/APRIL, proliferation-induced ligand; TP53, tumor protein p53; EPHB2, EPH receptor B2; IL-10, interleukin-10; IL-8, interleukin-8; NF-кB3, nuclear factor- $\kappa \mathrm{B} 3$; ROS1, c-ros oncogene 1 receptor tyrosine kinase; GnRH, gonadotropin-releasing hormone; CD40L, CD40 ligand; CD40, CD40 molecule; BAFF, B-cell-activating factor belonging to the TNF family; RANKL, receptor activator for nuclear factor- $\kappa \mathrm{B}$ ligand; JAK, janus kinase; STAT, signal transducing activator of transcription; MAPK, mitogen-activated protein kinase; NF- $\kappa \mathrm{B}$, nuclear factorkappaB; MAPK8, mitogen-activated protein kinase 8; TNF, tumor necrosis factor; IL-6, interleukin-6; VEGF, vascular endothelial growth factor; BATMAN-TCM, bioinformatics analysis tool for molecular mechanism of TCM; GO, gene ontology.

\section{Acknowledgment}

This work was supported by the Major Research Plan of Shandong Province (No. 2016GSF202041), National Natural Science Foundation of China (No. 81403080), Overseas Expertise Introduction Center for Discipline Innovation ("111 Center"; No. B16046) and Foundation for Innovative Research Groups of the National Natural Science Foundation of China (No. 81421005).

\section{Disclosure}

The authors report no conflicts of interest in this work.

\section{References}

1. Lerner A, Matthias T. Rheumatoid arthritis-celiac disease relationship: joints get that gut feeling. Autoimmun Rev. 2015;14(11):1038-1047.

2. Costenbader KH, Chang SC, Laden F, Puett R, Karlson EW. Geographic variation in rheumatoid arthritis incidence among women in the United States. Arch Intern Med. 2008;168:1664.

3. Markusse IM, Dirven L, Gerards AH, et al. Disease flares in rheumatoid arthritis are associated with joint damage progression and disability: 10-year results from the BeSt study. Arthritis Res Ther. 2015; 17(1):232.

4. Lindhardsen J, Ahlehoff O, Gislason GH, et al. The risk of myocardial infarction in rheumatoid arthritis and diabetes mellitus: a Danish nationwide cohort study. Ann Rheum Dis. 2011;70(6):929-934.

5. Burmester GR, Bijlsma JWJ, Cutolo M, McInnes IB. Managing rheumatic and musculoskeletal diseases - past, present and future. Nat Rev Rheumatol. 2017;13(7):443-448.

6. Obiri DD, Osafo N, Ayande PG, Antwi AO. Xylopia aethiopica (Annonaceae) fruit extract suppresses Freund's adjuvant-induced arthritis in Sprague-Dawley rats. J Ethnopharmacol. 2014;152(3):522-531.

7. Venkatesha SH, Berman BM, Moudgil KD. Herbal medicinal products target defined biochemical and molecular mediators of inflammatory autoimmune arthritis. Bioorgan Med Chem. 2011;19:21-29.

8. Peng C, Perera PK, Li Y, Fang W, Liu L, Li F. Anti-inflammatory effects of Clematis chinensis Osbeck extract(AR-6) may be associated with NF-KB, TNF- $\alpha$, and COX-2 in collagen-induced arthritis in rat. Rheumatol Int. 2012;32(10):3119-3125. 
9. Sun Y, Zhang X, Xie W, et al. Identification of UQCRB as an oxymatrine recognizing protein using a T7 phage display screen. J Ethnopharmacol. 2016;193:133-139.

10. Committee CP, editor. Pharmacopoeia of the People's Republic of China. Beijing: China Medical Science and Technology Press; 2015.

11. Pan T, Cheng TF, Jia YR, Li P, Li F. Anti-rheumatoid arthritis effects of traditional Chinese herb couple in adjuvant-induced arthritis in rats. J Ethnopharmacol. 2017;205:1-7.

12. Trame MN, Biliouris K, Lesko LJ, Mettetal JT. Systems pharmacology to predict drug safety in drug development. Eur J Pharm Sci. 2016;94:93-95.

13. Li S, Zhang B, Jiang D, Wei Y, Zhang N. Herb network construction and co-module analysis for uncovering the combination rule of traditional Chinese herbal formulae. BMC Bioinformatics. 2010;11:11.

14. Tao W, Xu X, Wang X, et al. Network pharmacology-based prediction of the active ingredients and potential targets of Chinese herbal Radix Curcumae formula for application to cardiovascular disease. J Ethnopharmacol. 2013;145(1):1-10.

15. Xu X, Zhang W, Huang C, et al. A novel chemometric method for the prediction of human oral bioavailability. Int J Mol Sci. 2012;13: 6964-6982.

16. Yue S, Liu J, Feng W, et al. System pharmacology-based dissection of the synergistic mechanism of Huangqi and Huanglian for diabetes mellitus. Front Pharmacol. 2017;8:694.

17. Yao Y, Zhang X, Wang Z, et al. Deciphering the combination principles of Traditional Chinese Medicine from a systems pharmacology perspective based on Ma-huang Decoction. J Ethnopharmacol. 2013;150(2):619-638

18. Kim K, Lee I, Gu W, Hyam SR, Kim D. $\beta$-Sitosterol attenuates high-fat diet-induced intestinal inflammation in mice by inhibiting the binding of lipopolysaccharide to toll-like receptor 4 in the NF- $\mathrm{BB}$ pathway. Mol Nutr Food Res. 2014;58(5):963-972.

19. Bin SM, Ameen SS. Beta-sitosterol: a promising but orphan nutraceutical to fight against cancer. Nutr Cancer. 2015;67(8):1214-1220.

20. Liu Y, Lei L, Wang X, et al. Plasma cholesterol-raising potency of dietary free cholesterol versus cholesteryl ester and effect of $\beta$-sitosterol. Food Chem. 2015;169:277-282.

21. Lee NY, Chung KS, Jin JS, Lee YC, An HJ. The inhibitory effect of nodakenin on mast-cell-mediated allergic inflammation via downregulation of NF- $\mathrm{KB}$ and Caspase-1 activation. $J$ Cell Biochem. 2017;118(11):3993-4001.

22. Burmester GR, Pope JE. Targeted treatments for rheumatoid arthritis 2 Novel treatment strategies in rheumatoid arthritis. Lancet. 2017;389: 2338-2348.

23. Nakahara H, Song J, Sugimoto M, et al. Anti-interleukin-6 receptor antibody therapy reduces vascular endothelial growth factor production in rheumatoid arthritis. Arthritis Rheum. 2003;48:1521-1529.

24. Otsuka T, Thacker JD, Hogge DE. The effects of interleukin 6 and interleukin 3 on early hematopoietic events in long-term cultures of human marrow. Exp Hematol. 1991;19:1042-1048.

25. Yoshitake F, Itoh S, Narita H, Ishihara K, Ebisu S. Interleukin-6 directly inhibits osteoclast differentiation by suppressing receptor activator of NF-kappaB signaling pathways. J Biol Chem . 2008;283:11535-11540.

26. Tanaka T, Narazaki M, Ogata A, Kishimoto T. A new era for the treatment of inflammatory autoimmune diseases by interleukin- 6 blockade strategy. Semin Immunol. 2014;26:88-96.

27. Hutchins AP, Poulain S, Miranda-Saavedra D. Genome-wide analysis of STAT3 binding in vivo predicts effectors of the anti-inflammatory response in macrophages. Mol Immunol. 2015;64(1):90-98.
28. Schett G, Tohidast-Akrad M, Smolen JS, et al. Activation, differential localization, and regulation of the stress-activated protein kinases, extracellular signal-regulated kinase, c-JUN N-terminal kinase, and p38 mitogen-activated protein kinase, in synovial tissue and cells in rheumatoid arthritis. Arthritis Rheum. 2000;43(11):2501-2512.

29. Sundarrajan M, Boyle DL, Chabaud-Riou M, Hammaker D, Firestein GS. Expression of the MAPK kinases MKK-4 and MKK-7 in rheumatoid arthritis and their role as key regulators of JNK. Semin Immunol. 2014;26(1):88-96.

30. Seyler TM, Park YW, Takemura S, et al. BLyS and APRIL in rheumatoid arthritis. J Clin Invest. 2005;115(11):3083-3092.

31. Nguyen HN, Noss EH, Mizoguchi F, et al. Autocrine loop involving IL-6 family member LIF, LIF receptor, and STAT4 drives sustained fibroblast production of inflammatory mediators. Immunity. 2017; 46(2):220-232.

32. Liu H. The Role of EphB/ephrinB in Inflammation. [dissertation]. 2013. University of Hedelberg.

33. Tak PP, Zvaifler NJ, Green DR, Firestein GS. Rheumatoid arthritis and p53: how oxidative stress might alter the course of inflammatory diseases. Immunol Today. 2000;21(2):78-82.

34. Pope RM. Apoptosis as a therapeutic tool in rheumatoid arthritis. Nat Rev Immunol. 2002;2(7):527-535.

35. Wang H, Ni S, Yang L. Expression of immune-related cytokine IL-1 $\beta$ in non-neoplastic epithelial disorders of the vulvar tissue and its clinical significance. Int J Clin Exp Pathol. 2017;10(5):4988-5000.

36. Davies KD, Doebele RC. Molecular pathways: ROS1 fusion proteins in cancer. Clin Cancer Res. 2013;19(15):4040-4045.

37. Bauch A, Superti-Furga G. Charting protein complexes, signaling pathways, and networks in the immune system. Immunol Rev. 2006;210(1):187-207.

38. Mayadas TN, Cullere X. Neutrophil $\beta 2$ integrins: moderators of life or death decisions. Trends Immunol. 2005;26(7):388-395.

39. Lee MS, Kim Y. Signaling pathways downstream of pattern-recognition receptors and their cross talk. Annu Rev Biochem. 2007;76:447-480.

40. Noort AR, Tak PP, Tas SW. Non-canonical NF- $\kappa B$ signaling in rheumatoid arthritis: Dr Jekyll and Mr Hyde? Arthritis Res Ther. 2015; 17(1): 15 .

41. Umicevic Mirkov M, Cui J, Vermeulen SH, et al. Genome-wide association analysis of anti-TNF drug response in rheumatoid arthritis patients. Ann Rheum Dis. 2013;72:1375-1381.

42. Banerjee S, Biehl A, Gadina M, Hasni S, Schwartz DM. JAK-STAT signaling as a target for inflammatory and autoimmune diseases: current and future prospects. Drugs. 2017;77(5):521-546.

43. Boyle DL, Soma K, Hodge J, Kavanaugh A, Mandel D. Extended report: the JAK inhibitor tofacitinib suppresses synovial JAK1-STAT signalling in rheumatoid arthritis. Ann Rheum Dis. 2015;74:1311-1316.

44. Elshabrawy HA, Chen Z, Volin MV, Ravella S, Virupannavar S, Shahrara S. The pathogenic role of angiogenesis in rheumatoid arthritis. Angiogenesis. 2015;18(4):433-448.

45. Carmeliet P. Angiogenesis in life, disease and medicine. Nature. 2005;438(7070):932-936.

46. Olsson A, Dimberg A, Kreuger J, Claesson-Welsh L. VEGF receptor signalling - in control of vascular function. Nat Rev Mol Cell Bio. 2006;7(5):359-371.

47. Li W, Liang RR, Zhou C, et al. The association between expressions of Ras and CD68 in the angiogenesis of breast cancers. Cancer Cell Int. 2015;15(1):17.

48. Biniecka M, Kennedy A, Fearon U, Ng CT, Veale DJ. VEGF as an activity marker in rheumatoid arthritis. Int J Clin Rheumatol. 2010; 5(3):287-289. 


\section{Publish your work in this journal}

Drug Design, Development and Therapy is an international, peerreviewed open-access journal that spans the spectrum of drug design and development through to clinical applications. Clinical outcomes, patient safety, and programs for the development and effective, safe, and sustained use of medicines are the features of the journal, which has also been accepted for indexing on PubMed Central. The manuscript management system is completely online and includes a very quick and fair peer-review system, which is all easy to use. Visit http://www.dovepress.com/testimonials.php to read real quotes from published authors.

Submit your manuscript here: http://www.dovepress.com/drug-design-development-and-therapy-journal 